\title{
Una aproximación a la clasificación de las universidades chilenas
}

\author{
An approach toward a classification of Chilean higher education institutions \\ Emilio Rodríguez-Ponce ${ }^{1} \quad$ Héctor Gaete Feres ${ }^{2} \quad$ Liliana Pedraja-Rejas $^{3}$ \\ Carmen Araneda-Guirriman ${ }^{4}$
}

Recibido 28 de julio de 2014, aceptado 19 de marzo de 2015

Received: July 28, 2014 Accepted: March 19, 2015

\begin{abstract}
RESUMEN
La sociedad del conocimiento y la globalización han impactado a la educación superior generando varios cambios en las últimas décadas. Estos cambios hacen referencia a un aumento en la demanda, la masificación de la matrícula y la diversificación de las instituciones de educación superior, con o sin fines de lucro, además de un incremento en la presencia de universidades de origen privado. En consecuencia las universidades presentan diferentes misiones, formando graduados con una calidad variable. El aseguramiento de la calidad, por tanto, se visualiza como un elemento crucial para resguardar la calidad de la formación académica de los estudiantes. En este sentido, se busca generar una clasificación de las universidades, para ello se efectúa un ordenamiento espacial en un gráfico de dispersión de 52 universidades chilenas, utilizando para este fin las variables de acreditación institucional y de rentabilidad sobre el patrimonio, usando además las medias de ambas. Ambos aspectos conciben en la conformación de cuatro grupos de universidades, generando así una nueva aproximación para el análisis de las universidades existentes en Chile.
\end{abstract}

Palabras clave: Universidades chilenas, calidad, rentabilidad, educación superior, privatización.

\begin{abstract}
The knowledge society and globalization have impacted higher education, generating a series of changes in recent decades. These changes make reference to the growing demand, widespread growth of enrollment and diversification of higher education institutions, with or without profit, in addition to a large increase in the presence of universities from private sources. Therefore, universities have different missions, thus forming graduates with heterogeneous quality. The quality assurance, therefore, is seen as crucial to safeguard the quality of the academic training of students. In this sense, the aim of this paper is to generate a ranking of universities, for which a spatial arrangement of 52 Chilean universities is carried out in a scatter plot, using for this purpose the variables of institutional accreditation and return of equity, besides using the average of both. These two aspects conceive in forming four groups of universities, thus generating a new approach for the analysis of existing universities in Chile.
\end{abstract}

Keywords: Chilean universities, quality, profitability, higher education, privatization.

\footnotetext{
1 Instituto de Alta Investigación. Universidad de Tarapacá. Avenida General Velásquez No 1775 . Arica, Chile. E-mail: emilior.rodriguez.ponce@gmail.com

2 Universidad del Bío-Bío. Avenida Collao N 1202. Concepción, Chile. E-mail: hgaete@ubiobio.cl

3 Centro de Estudios Universidad de Tarapacá, CEUTA. Avenida 18 de septiembre N ${ }^{\circ} 2222$. Arica, Chile. E-mail: lpedraja@uta.cl

4 Escuela de Ingeniería Industrial, Informática y de Sistemas. Universidad de Tarapacá. Avenida 18 de septiembre $\mathrm{N}^{\circ} 2222$. Arica, Chile. E-mail: caraneda@uta.cl
} 


\section{INTRODUCCIÓN}

La globalización y la sociedad del conocimiento han generado un nuevo contexto para la educación superior, lo que ha conllevado a varios cambios. En primera instancia, se puede hacer referencia a un aumento en la demanda por acceder a la educación terciaria y a una masificación de esta a causa de la importancia que tiene hoy la formación profesional para la sociedad. Asimismo, ha ido en aumento la proliferación de instituciones de educación superior de diversa índole, es decir, pública, privada, con y sin fines de lucro, existiendo, por tanto, un sistema mixto de educación terciaria.

Asimismo, el sector de la educación superior, durante muchos años, ha jugado un rol clave en el desarrollo de diferentes países, lo que lleva a asumir que ha tenido gran importancia como conductor de la economía global [40].

Es posible establecer, por consiguiente, que este incremento por la demanda de educación superior ha resultado en una diversidad de proveedores que entregan educación alrededor del mundo [24]. Junto a esta diversificación del sistema de educación terciaria se ha incrementado la educación superior con fines de lucro durante los recientes años [27, 29], esto genera preocupación, ya que estas organizaciones han venido a jugar un rol más prominente en la educación terciaria de América Latina, existiendo inquietud por posible fraude y otras prácticas cuestionables, que usualmente involucran la ayuda estatal para estudiantes y el reclutamiento de estos [4].

Es factible destacar además que existe un desdibujamiento de los límites entre las instituciones públicas y privadas, pues muchas universidades públicas ahora encuentran necesario buscar financiamiento privado y cobran por concepto de matrícula o servicio. En muchos otros países, las instituciones privadas pueden optar a fondos públicos y participar en actividades sociales sin fines de lucro [24]. Por tanto, el aseguramiento de la calidad en este escenario se configura como una herramienta fundamental al momento de analizar la variedad de instituciones de educación superior existentes, en este caso particular, en Chile. Más aún al considerar los roles que tienen las instituciones de educación superior en la sociedad del conocimiento en lo que respecta a la creación de conocimiento avanzado, difusión de conocimiento y contribución al desarrollo y equidad. En este sentido, la calidad se configura como el marco contextual de estas acciones, al establecer mecanismos para asegurar la idoneidad de la provisión de los servicios académicos y garantizar el valor público de los títulos y grados entregados por las universidades [47]. A este respecto, el desarrollo del aseguramiento de la calidad informa de pasos decididos hacia la generación de notificaciones respecto de la calidad de la educación superior que, a pesar de que posee problemas y asimetrías, sirve a instituciones, estudiantes, usuarios y a los fines de la regulación pública [48].

Junto a todos estos cambios que ha experimentado la educación superior durante las últimas décadas se ha ido incrementando la preocupación por la calidad de las instituciones de educación superior, debido al impacto que tienen las prácticas de aseguramiento de la calidad en los resultados alcanzados por estas.

De este modo, los gobiernos de gran parte del mundo han incorporado en sus agendas de educación superior de las últimas décadas los temas de aseguramiento y mejoramiento de la calidad, configurándose como un importante foco de atención [41].

Con el fin de generar una primera aproximación conducente a la clasificación de las universidades chilenas, se realiza un ordenamiento en torno a la rentabilidad sobre el patrimonio y la calidad institucional de dichas instituciones, utilizando como criterio las medias de ambos aspectos. Al mismo tiempo se debe precisar que el estudio es de tipo transeccional con datos del 2011. Este año fue clave para la coyuntura de educación universitaria nacional, ya que por primera vez las instituciones de educación superior transparentaron información referida a su patrimonio.

Con referencia a ello, se puede establecer que durante el 2011 el país experimentó una crisis del sistema de educación, principalmente de la educación, iniciándose un debate nacional al respecto que perdura hasta la actualidad. En relación con este movimiento se puede establecer que dicha situación puede ser entendida a causa de una crisis en la legitimidad de la clase política que ha conllevado a una crítica del modelo educativo 
general, pues este no ha traído, desde la perspectiva de la sociedad, ni la igualdad ni la integración, sino que ha perpetuado las diferencias sociales [12]. En efecto, es la misma ciudadanía la que se muestra crítica frente al modelo educativo que reproduce y perpetúa la desigualdad social, trayendo a discusión aspectos como el financiamiento, el lucro, el acceso y la calidad de la educación superior, entre otros [43].

Los resultados del estudio muestran claramente que las instituciones de educación superior analizadas se ordenan en grupos caracterizados de la siguiente forma: universidades con alta acreditación y baja rentabilidad; grupo 2: universidades con alta acreditación y alta rentabilidad; grupo 3: universidades con baja acreditación y baja rentabilidad y grupo 4: universidades con baja acreditación y alta rentabilidad.

\section{MARCO TEÓRICO}

\section{Privatización de la educación superior}

Una de las tendencias más claras en la educación superior del mundo entero ha sido el crecimiento de la educación superior privada, con y sin fines de lucro $[44,49,55,30]$. En Europa, la educación superior privada emerge en el periodo de la post guerra, antes de su espectacular crecimiento en los expaíses comunistas, después de 1989 [26].

El incremento de la demanda por educación superior ha resultado en una diversidad de proveedores que entregan educación superior, pueden clasificarse en dos tipos de categorías: las tradicionales instituciones de educación superior orientadas, normalmente, a la docencia, la investigación y el servicio a la sociedad; y las nuevas alternativas de proveedores, focalizados en la docencia y la entrega de servicios educativos, usualmente sobre una base comercial [24]. En el caso de Australia, por ejemplo, el aumento de la educación superior privada se vincula a factores externos, como la masificación de la educación superior, la elección de los estudiantes, la demanda no satisfecha, el movimiento estudiantil, la información disponible al público respecto de la clasificación de la universidad y la introducción del movimiento de calidad [51]. Este aumento en la demanda por la educación superior se ha traducido en una mayor proliferación de instituciones privadas con y sin fines de lucro.
A este respecto, de acuerdo con una estimación, el $30 \%$ de la matrícula a nivel mundial en educación superior es ahora privada [5]. Esta cifra refleja que en el último tiempo ha habido una expansión de las instituciones de educación privadas, las que son en su mayoría con fines de lucro, y tienen como mayor fuente de ingreso la matrícula [54]. En consecuencia, esta proliferación de alternativas de educación superior trae consigo aspectos como el lucro en la educación postsecundaria, poniendo su foco de atención en la rentabilidad que le genera su quehacer habitual, por sobre su acción formadora de capital humano, siendo, por tanto, necesario poner atención en la calidad de los procesos académicos y formativos de estas organizaciones que ponen su énfasis en las acciones con fines comerciales.

A este respecto se puede establecer que históricamente las instituciones con y sin fines de lucro han sido vistas como diametralmente opuestas, con un énfasis en las variadas diferencias entre estas dos aproximaciones a la educación superior con pocas áreas que se reconocen como similares entre sí, junto con ello, durante la última década se ha visto un incremento del rol de los propietarios de instituciones de educación superior y la orientación empresarial que conduce a su crecimiento [33].

Chile, al igual que en el resto del mundo, ha experimentado la expansión y modernización del sistema de educación superior. En este contexto, a partir de 1981 el gobierno ha estimulado de manera activa el surgimiento del sector privado. Es más, la mayor proporción del aumento en la cobertura de educación terciaria desde ese año ha sido posible por el rápido crecimiento de las instituciones de educación superior privadas, financiadas en gran parte por los estudiantes y sus familias. De esta manera, el crecimiento del sector privado ha sido una característica de muchos países de América Latina, especialmente de Chile, donde se ha experimentado uno de los más rápidos aumentos de la región [39]. Cabe señalar, de acuerdo con los resultados de un estudio efectuado con las variables de financiamiento actual de las universidades del Consejo de Rectores de las Universidades Chilenas (CRUCH), confirma asimetrías y desigualdades entre las universidades, principalmente para las instituciones estatales de regiones en la entrega de recursos básico, resaltando el mayor aporte entregado a las universidades de mayor complejidad [2]. En efecto, la nueva 
institucionalidad de la educación superior chilena impuesta en 1981 estableció la diferenciación entre las universidades creadas antes de la ley, como las estatales o aquellas privadas con carácter público, y aquellas generadas a partir de ese momento, las instituciones privadas, derivándose a partir de esto distinciones respecto del régimen de la propiedad estatal/privada [37].

En consecuencia, en Chile se generó un modelo de educación superior que, de acuerdo con las palabras de Lavados Montes [28], se caracteriza por considerar que la educación y el conocimiento son bienes que pueden ser transados en el "mercado del conocimiento" (p. 23), donde el pago de aranceles y por prestaciones de servicios son expresiones concretas de este concepto. Asimismo, señala que es el mercado quien por medio de la competencia regula la calidad y la pertinencia del sistema de educación superior y de sus integrantes. Además del hecho que ciertas actividades y procesos académicos deben ser subvencionados, para lograr el desarrollo de actividades y la equidad e igualdad de oportunidades de acceso para los estudiantes. En consecuencia, esta visión supone que la educación y el conocimiento se configuran en primera instancia como bienes que pueden ser transados en el mercado.

Como se señalaba con anterioridad, en gran parte de los países desarrollados la población joven ha incrementado en número y consecuentemente ha aumentado la demanda por educación superior, sin embargo, los gobiernos no pueden responder a todas las demandas. Por consiguiente, desarrollaron el sector de educación superior privado como una alternativa de solución [21]. Esta es una de las estrategias utilizadas para diversificar las formas en que la educación terciaria es ofrecida y financiada, teniendo, en consecuencia, ahora el más rápido crecimiento en el sector de educación postsecundaria alrededor del mundo [11]. Siendo aún más específicos, si se limitara a las instituciones legales con fines de lucro este sector podría ser pequeño, pero está creciendo, como ocurre con el caso de Brasil, donde en el 2005 las instituciones universitarias con fines de lucro legales alcanzaron $19 \%$ del total de la matrícula de educación superior, no mucho más abajo que el sector público [31]. Se puede establecer que la educación superior con fines de lucro se ha ido convirtiendo en una alternativa para quienes quieren acceder a la educación superior.
Es así como esta situación puede considerarse como el resultado de un proceso que fomenta la corporatización, la diversificación y la masificación, dando lugar a la creación de nuevas instituciones de educación superior, siendo muchas de ellas etiquetadas como proveedoras privadas o con fines de lucro. En efecto, el sector de la educación superior privada prosperó, introduciéndose muchas nuevas facultades y programas académicos vinculados con las demandas actuales de la industria [52].

Asimismo, de acuerdo con los planteamientos de Tilak [54], este rápido crecimiento de la educación superior en los últimos años está asociado con unas importantes características y problemas. Como primer punto se puede señalar que hay un salto significativo desde la filantropía hacia la rentabilidad en la creación de instituciones privadas. Segundo, los sistemas de educación superior en los países en vías de desarrollo son más privatizados que en los países desarrollados. Tercero, en muchos países las universidades privadas cuentan con un gran número de universidades totales, pero poseen un número pequeño de matrículas. Cuarto, la educación superior privada se ve ensombrecida por asuntos como las tasas, la autonomía, la equidad, la excelencia académica y la pertinencia.

Se debe establecer que la ley no es clara acerca de cuáles instituciones son con fines de lucro y sin fines de lucro, incluso, los expertos en un mismo país no siempre están de acuerdo respecto de si los fines de lucro están permitidos [31]. Esta situación puede conducir a dificultades de identificación por parte de quienes quieren acceder a la educación superior.

La privatización en la educación superior significa muchas cosas, mientras que la universidad pública es considerada generalmente como una institución financiada por y en respuesta al gobierno local, provincial o nacional, las instituciones privadas no reflejan un modelo consistente. Las instituciones privadas pueden operar completamente con activos privados o parcialmente con fondos públicos, podrían ser además con fines de lucro o sin fines de lucro, así como también podrían ser capaces de rendir cuenta al gobierno u operar completamente fuera de la regulación local. Dichas instituciones podrían tener propietarios o inversores, o funcionar como una fundación [1]. De esta forma, en Estados 
Unidos las instituciones de educación superior con fines de lucro suelen ser dirigidas por profesionales del área de los negocios altamente calificados, pero con una experiencia académica limitada [8]. Lo anterior refleja que el centro de preocupación de las universidades con fines de lucro no es académico, sino que es la generación de excedentes de sus actividades, generando un desafío para el resguardo $\mathrm{y}$ aseguramiento de la calidad de las instituciones de educación superior.

A este respecto se puede señalar que desde 1975 las universidades se han vuelto mucho más agresivas en el intento por hacer dinero de sus actividades de investigación y educativas. Muchas instituciones han iniciado notables programas de licencia de patentes, emprendimientos con fines de lucro en educación por internet, junto con una amplia variedad de otras iniciativas comerciales [6]. Convirtiéndose en consecuencia en una organización cuyo propósito fundamental es satisfacer las necesidades y deseos de los clientes y obtener ganancias, siendo esta la rentabilidad del cliente la que conducirá exitosamente la gestión de una empresa en el largo plazo [25]. Vinculado a esto se encuentra el aumento de la aplicación del marketing en la esfera de la educación superior y su orientación a los clientes por la rentabilidad [25]. De esta forma, la rentabilidad es un aspecto de preocupación central de algunas instituciones de educación superior, siendo muchas veces más importante que el propio quehacer académico o su función formadora de capital humano de calidad.

\section{Calidad en la educación superior}

La preocupación por la calidad y sus estándares no es nueva, ya que desde mediados de la década de los ochenta se inicia el debate acerca de los sistemas de educación superior. El interés público por la calidad fue intensificado por el incremento de la atención proporcionada por el gobierno inglés a reformar la educación superior [14]. Durante la década de los noventa la calidad evolucionó desde una posición marginal hacia una preocupación central en la educación terciaria, junto con los problemas de financiamiento. En efecto, la calidad ha sido una de las mayores preocupaciones de la educación superior durante los años 90 [17]. La calidad de la educación superior y la necesidad de contar con un mecanismo eficiente de aseguramiento de la calidad va más allá de las instituciones, convirtiéndose en un tema prioritario de la estrategia de educación superior [23].

En efecto, los sistemas de aseguramiento de la calidad se han convertido en la herramienta cada vez más dominante en la gestión de los sectores de educación superior, estimándose que aproximadamente la mitad de los países en el mundo cuenten con un sistema de aseguramiento de la calidad u organismos que regulen la calidad de la educación superior [22].

Si se tiene una mirada país, particularmente a nivel de países emergentes, se observa que la ambición de los ministerios de educación es entregar un amplio acceso a la educación superior de alta calidad y a un bajo costo [11]. De acuerdo con Cheng [9], la calidad en la educación es la característica de un conjunto de elementos de entrada, procesos, y resultados del sistema educativo que entrega servicios que logran satisfacer completamente a los grupos estratégicos, tanto internos como externos, mediante el cumplimiento de sus expectativas explícitas e implícitas.

Ahora bien, a nivel institucional, un sistema de aseguramiento y gestión de calidad debiera estar estrechemante ligado a la calidad del diseño estratégico que se genere en la propia organización [45].

Para Harvey y Green [18] existen cinco aproximaciones de calidad identificables relacionadas con la educación. La primera de ellas considera a la calidad como excepción, donde se ve a la calidad como algo especial, tradicionalmente asociado este concepto a algo distintivo y elitista, y en términos educacionales está vinculado a las nociones de excelencia, alta calidad, inalcanzable para la mayoría. En la segunda aproximación la calidad es vista como perfección, es decir, la calidad como un resultado consistente o sin defectos, en este sentido, democratiza la noción de calidad, pues si la consistencia puede ser lograda la calidad puede ser, entonces, alcanzada por todos. La tercera, visualiza la calidad como aptitud para el logro de objetivos, considerando a la calidad en términos de la satisfacción de los requerimientos del consumidor, necesidades o deseos, quien teóricamente los especifica. En educación aptitud para el logro de los objetivos está usualmente basado en la habilidad de una institución para cumplir su misión o programa de estudio y sus metas a cabalidad. La 
cuarta aproximación considera la calidad como valor por dinero, visualizando a la calidad en términos de retorno sobre la inversión. Si el mismo resultado puede ser alcanzado a un menor costo, o mejores resultados pueden ser alcanzados al mismo costo, entonces el cliente tiene un producto de calidad. El aumento de esta tendencia hace necesaria para los gobiernos la rendición de cuentas por parte de la educación superior, que reflejen una aproximación a la relación calidad-costo. Finalmente, la calidad como transformación es una clásica noción de calidad, que la configura en términos de cambio de un estado a otro. En términos educacionales, la transformación se refiere al realce y empoderamiento de los estudiantes o el desarrollo de un nuevo conocimiento.

Por tanto, se puede establecer que la calidad es un concepto multidimensional que no puede ser sustentado por un solo indicador [10]. Es así como la definición que realiza Harvey y Green [17] logra una visión global y completa de los aspectos.

Vinculado con lo anterior, se debe considerar que la calidad de servicio, con énfasis en la satisfacción de los estudiantes, está emergiendo como un nuevo campo de preocupación. Esto, porque en orden de atraer más estudiantes, atender sus necesidades y retenerlos, los proveedores de educación se están involucrando en entender a los estudiantes y sus percepciones de la calidad de servicio [38].

El crecimiento que ha experimentado la educación superior transnacional ha sido asociado con el crecimiento de la preocupación relacionada a la calidad y ha incrementado la dificultad de gestionar la calidad de los distintos grados de transnacionalismo y proveedores [32]. En consecuencia, la calidad es un tema de preocupación permanente para los gobiernos y autoridades académicas, en particular debido a su contingencia y capacidad de estar presente como un aspecto relevante en los cambios ocurridos y manifestados en la educación superior.

\section{METODOLOGÍA}

La metodología del estudio es de tipo cuantitativa, tiene un propósito descriptivo y es de carácter transaccional, ya que se trabaja con los datos financieros entregados por 52 instituciones de educación superior. Siendo este el único año en el que la mayoría de las universidades entregaron dicha información. De esta forma, se trabajó con una muestra de 52 universidades estatales y privadas caracterizadas por haber participado en los procesos de acreditación institucional de la Comisión Nacional de Acreditación de Chile y que cuentan con información financiera del Ranking de Universidades de la Revista América Economía del 2011.

Las variables utilizadas son la calidad institucional, medida por medio de los años de acreditación institucional otorgados por la Comisión Nacional de Acreditación de Chile, cuya información fue obtenida de la página web de CNA Chile (al 31 de julio del 2012). Este proceso de medición de la calidad se efectúa mediante el número de años de acreditación institucional concedidos por una institución autónoma externa como lo es la Comisión Nacional de Acreditación, la que utiliza como antecedentes el informe de autoevaluación y el Informe de los Pares Evaluadores, siendo considerada por investigaciones anteriores como un proxy razonable para medir la calidad de las universidades chilenas [46].

La variable rentabilidad de las universidades se obtiene desde los datos financieros recolectados a partir de la siguiente fórmula:

$$
\left[\frac{\text { Utilidad }}{\text { Patrimonio }}\right] 100
$$

Asimismo, para el cálculo del patrimonio se consideró la diferencia entre los activos y pasivos de la universidad.

Con el objetivo de clasificar de manera visual las universidades de acuerdo con las dos variables señaladas anteriormente se elaboró un gráfico de dispersión para observar cómo se ordenaban y distribuían los casos en torno a las variables señaladas (ver Figura 1). Posteriormente se calcularon las medias de ambas variables con el fin de establecer un punto de corte en la distribución de los casos.

\section{RESULTADOS Y DISCUSIÓN}

En primera instancia, vale precisar que la clasificación obtenida en este estudio responde a los datos obtenidos en un contexto de tiempo determinado (2011-2012), en consecuencia, para analizar en 


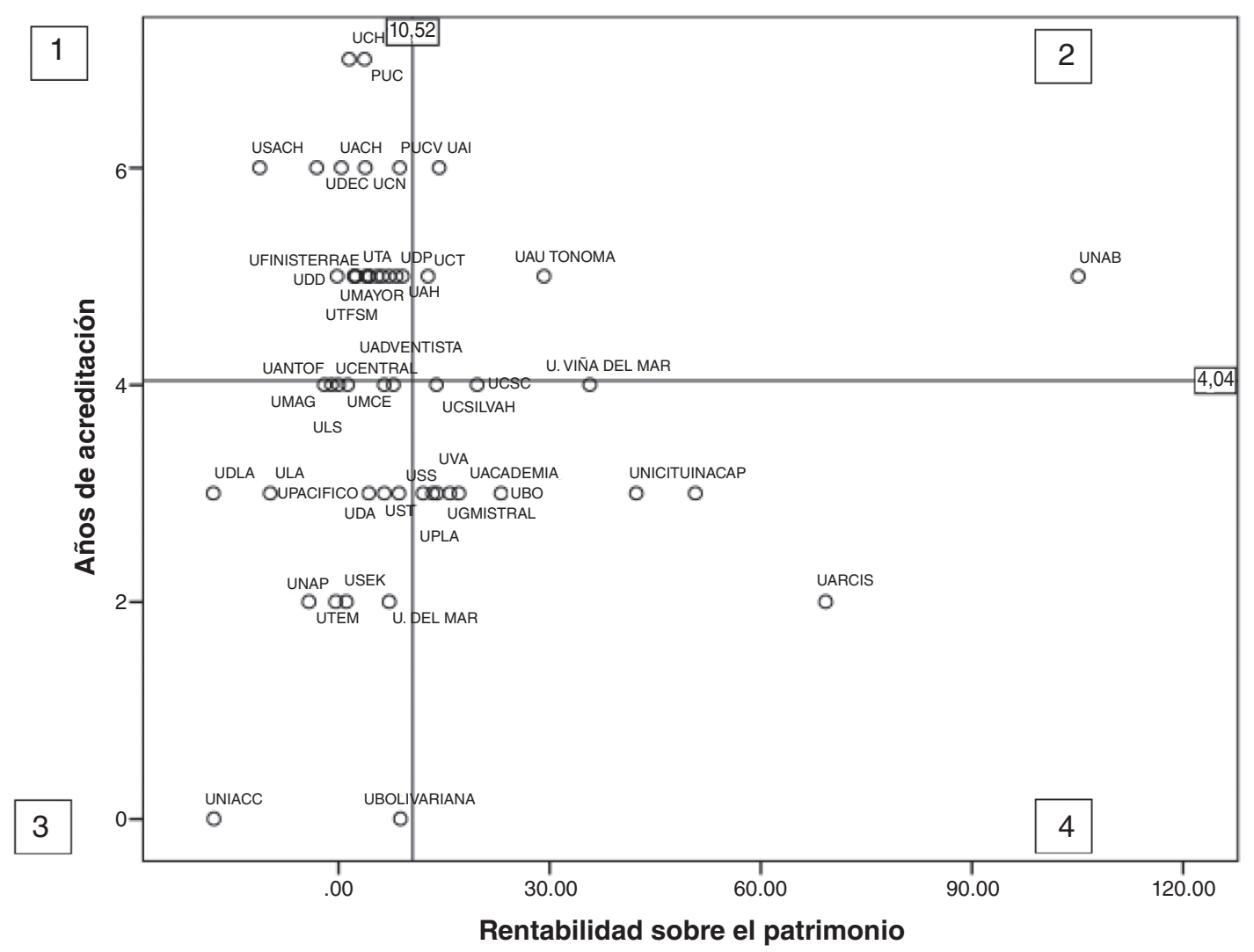

Figura 1. Dispersión de los casos de acuerdo con los años de acreditación institucional y rentabilidad sobre el patrimonio.

Fuente: Elaboración propia.

mayor profundidad si estos resultados son una tendencia o no, se requiere de nuevos análisis para otros periodos con las variables correspondientes.

Al realizar el gráfico de dispersión con las variables de acreditación institucional y rentabilidad de las universidades es posible observar la forma en que se agrupan los casos, esto debido al cálculo de las medias de ambas variables, que permiten generar un corte en la distribución y visualizar claramente cuatro grupos. En efecto, al analizar la Figura 1 se puede observar que la media para los años de acreditación es de 4,04, y la media de rentabilidad sobre el patrimonio es de $10,51 \%$.

A partir de lo anterior, se puede identificar un primer cuadrante donde se encuentran universidades con alta acreditación y baja rentabilidad. En este segmento es posible identificar a un conjunto de universidades caracterizadas por estar sobre el promedio de 4,04 años de acreditación institucional, y bajo el promedio de rentabilidad sobre el patrimonio de $10,5 \%$. Esto quiere decir que son instituciones orientadas principalmente a su quehacer académico, estando direccionadas a la docencia de pre y postgrado, la investigación y la vinculación con el medio, correspondientes principalmente a instituciones estatales y tradicionales de educación superior, estas rechazan el hecho de que el lucro haya reemplazado del centro valores como la libertad académica al implementar políticas diseñadas para incrementar la producción de utilidades [53]. Por tanto, estas universidades ganan menos que el mercado y tienen una alta calidad.

Vale precisar que es posible encontrar al menos seis casos de instituciones que son privadas que requerirían de un posterior análisis en profundidad, 
ya que al menos dos universidades privadas poseen empresas relacionadas que pueden estar logrando altos niveles de rentabilidad para los propietarios por una vía indirecta. Las universidades que se encuentran en este cuadrante son: Pontificia Universidad Católica de Chile, Universidad de Chile, Universidad Católica del Norte, Universidad Austral, Universidad de Concepción, Universidad de Santiago, Universidad Alberto Hurtado, Universidad de Tarapacá, Universidad Diego Portales, Universidad Mayor, Universidad Técnica Federico Santa María, Universidad Finis Terrae, Univesidad de los Andes, Universidad Católica del Maule, Universidad de la Frontera, Universidad del Bío-Bío, Universidad de Talca y Universidad del Desarrollo.

El segundo cuadrante, en tanto, se encuentra denominado por universidades que presentan una alta acreditación y también una alta rentabilidad. En efecto, agrupa a universidades que se ubican por sobre los promedios de ambas variables. Dichos casos se caracterizan por ser instituciones esencialmente privadas. A este respecto es posible señalar que ha habido un rápido crecimiento de la educación superior privada, teniendo alrededor de un tercio de la matrícula alrededor del mundo $[30,11,42]$. Chile no ha estado exento de esta realidad, presentando un importante número de instituciones privadas.

Estos resultados demuestran que dentro de esta diversidad de universidades es posible encontrar instituciones que se ubican sobre los promedios de años de acreditación y de rentabilidad sobre el patrimonio, caracterizadas por obtener, además de ganancias, calidad en su función educativa.

Las instituciones universitarias que se encuentran en este grupo son las siguientes: Universidad Adolfo Ibáñez, Universidad Autónoma, Universidad Católica de Temuco y la Universidad Andrés Bello.

En este sentido, Brunner y Uribe [7] plantean que en el nuevo sistema universitario coexisten instituciones de distinta naturaleza, que mezclan en diferentes proporciones y grados la orientación al medio o al mercado y la disposición a las disciplinas que cultivan, universidades que compatibilizan el fin del lucro con las actividades propias de la enseñanza, entre otras características. Por tanto, continuando con los autores, la experiencia muestra que el fenómeno de la masificación de la educación superior conlleva a una vasta variedad de fisonomías universitarias, existiendo por tal razón diversos modelos de universidad.

No obstante, se debe precisar que en Chile el lucro en la educación superior se encuentra prohibido, como lo demuestra lo dispuesto en el Decreto con Fuerza de Ley $\mathrm{N}^{\circ} 1$ del 30 de diciembre de 1980, en el punto IV, acerca de la creación y disolución de universidades, donde el artículo 15 indica lo siguiente: "Podrán crearse universidades, las que deberán constituiré como personas jurídicas de derecho privado sin fines de lucro" $[52,9]$.

Vinculado con lo indicado anteriormente es posible señalar que investigadores han abogado que el incremento en los ingresos y la rentabilidad por medio de la mejora de la calidad se logran principalmente porque una mejor calidad conduce a la satisfacción del cliente [3]. Es decir, la calidad en estos casos podría estar repercutiendo en la rentabilidad alcanzada por dichas instituciones.

No obstante, en contraposición a este planteamiento es posible establecer que las instituciones de educación superior privadas han sido criticadas por su carencia de calidad y acceso [15], situación que se puede aplicar a los casos ubicados en los cuadrantes 3 y 4, donde en el primer caso se encuentran las universidades que están bajo el promedio de años de acreditación. De esta forma, es posible señalar que el tercer cuadrante identificado se encuentra conformado por universidades con bajas acreditación y rentabilidad. Se trata de instituciones principalmente privadas, recientes y de bajo posicionamiento de la marca, aunque es posible encontrar ocho casos de universidades estatales, las que se encuentran bajo el promedio de años de acreditación institucional y que además muestran una baja rentabilidad. En el caso de las universidades públicas se trata de instituciones públicas emergentes, o que han tenido problemas de gestión o manejo operacional en los años recientes. Las instituciones pertenecientes a este segmento son: Universidad Central, Universidad Adventista, Universidad Metropolitana de Ciencias de la Educación, Universidad de Antofagasta, Universidad de Magallanes, Universidad de La Serena, Universidad San Sebastián, Universidad de Atacama, Universidad del Pacífico, Universidad de las Américas, Universidad de Los Lagos, Universidad del Mar, Universidad Internacional SEK, Universidad 
Arturo Prat, Universidad Tecnológica Metropolitana, Universidad Metropolitana, y Universidad de Artes, Ciencias y Comunicación.

Por último, es posible encontrar que el cuarto cuadrante se encuentra conformado por universidades con alta rentabilidad y baja acreditación. En este grupo es posible encontrar instituciones esencialmente privadas que logran rentabilizar sobre el mercado, incluyendo dos casos de universidades estatales, las que poseen escasa acreditación por problemas recientes de gestión.

Igual que en el caso anterior, las universidades ubicadas bajo la media de años de acreditación institucional y sobre la media de rentabilidad se caracterizan por la baja calidad de su quehacer académico.

Esta situación se vincula con el crecimiento de la educación privada en muchas partes del mundo, lo que ha expresado su preocupación por la calidad y el nivel educacional entregado en dichas instituciones [50]. Esto, esencialmente porque las instituciones privadas, como señalan Altbach, Reisberg y Rumbley [1], podrían rendir cuentas o también operar fuera de la regulación. Se puede deducir, de acuerdo con los planteamientos de este autor, que el sistema de regulación en ese sentido es bastante reducido.

La alta rentabilidad evidenciada en estos casos puede ser entendida en el sentido de que para gran parte de las naciones la educación superior es entendida como pública, no obstante, la primera excepción es Estados Unidos donde está la noción de que la educación superior es un mercado [34]. En efecto, se puede establecer que las universidades actúan como empresas, estas se encuentran satisfechas cuando los intercambios y las relaciones resultan rentables o crean valores económicos de los clientes [19].

Relacionado con esto se encuentra la preocupación existente de parte de los estudiantes de instituciones privadas de China, quienes de acuerdo con recientes reportes han iniciado paros, protestas y disturbios en respuesta a las nuevas regulaciones respecto del grado que reciben. Algunas de estas protestas de los estudiantes están motivadas por las excesivas tarifas cobradas en las instituciones de educación privadas, así como también por las quejas asociadas a la calidad de la enseñanza y el estado de las titulaciones que se ofrecen después de la graduación, ya que algunos manifestantes señalan la disonancia entre las promesas hechas por estas instituciones acerca de la admisión y la realidad de la graduación [35]. Esto demuestra las dificultades y desafíos que debe enfrentar la educación superior en términos de calidad, al encontrarse frente a una diversidad de instituciones públicas y privadas que tienen al mismo tiempo diferentes niveles de calidad, valores de matrícula, y rentabilidad.

Las universidades que conforman este grupo son: Universidad Católica Silva Henríquez, Universidad Tecnológica de Chile INACAP, Universidad Iberoamericana de Ciencias y Tecnología, Universidad Bolivariana, Universidad Católica de la Santísima Concepción, Universidad Gabriela Mistral, Universidad de Playa Ancha, Universidad de Valparaíso, Universidad Santo Tomás, y Universidad de Arte y Ciencias Sociales.

En efecto, y en consideración a los resultados obtenidos, es posible establecer que hoy es cada vez más frecuente hacer referencia a la educación superior como un valor activo o cómo un rentable negocio se ha vuelto más común [13], demostrándose que Chile no está exento de esta realidad. En este sentido, quienes se oponen a la privatización mencionan que el sector trata de entregar programas especiales que obtengan mayores beneficios privados y ganancias [42]. La rentabilidad en las instituciones de educación terciaria se configura como un desafío para los procesos de aseguramiento de la calidad, los que en algunos casos se ven mermados por no contribuir de manera directa al incremento de las ganancias.

Es posible destacar que la generación de lucro al interior de la educación superior no es un hecho reciente, como sucede con algunas universidades norteamericanas que tradicionalmente han operado sobre bases sin fines de lucro, han comenzado a desarrollar programas educacionales que producirían un excedente que ellos podrían ocupar para otros propósitos [6]. De acuerdo con Mungu [36], esta orientación de mercado es posiblemente una ayuda a las instituciones de educación superior en su esfuerzo por superar los desafíos y la presión de los cambios en el ambiente.

Sin embargo, los detractores a la educación con fines de lucro critican el movimiento considerándolo 
en el fondo como incompatible con las nociones de bien público de la educación, junto a la certeza referente a la generación de víctimas en esta carrera por aumentar las ganancias. En este sentido, la educación con fines de lucro ha generado víctimas como la primera generación en acceder a la educación superior, la clase pobre y trabajadora y los estudiantes veteranos [20]. Asimismo, en esta carrera por aumentar las ganancias se descuida la calidad del quehacer académico, perjudicando a sus estudiantes y su futura inserción laboral. En consecuencia, el objetivo que debe primar es disponer de una educación superior de calidad, a la que puedan acceder todos los estudiantes.

\section{CONCLUSIONES}

La calidad institucional de las universidades junto a su rentabilidad sobre el patrimonio permite en conjunto efectuar una aproximación respecto de la clasificación de universidades, ya que ambas variables se configuran como indicadores de su quehacer y objetivo fundamental de su esencia como institución. Esta clasificación en torno a estas variables complementa la forma de analizar las instituciones de educación superior de Chile, enriqueciendo el análisis y la discusión al respecto.

En consecuencia, las universidades analizadas se clasifican y ordenan en cuatro conjuntos, donde dos de estos grupos se logran ubicar sobre la media de años de acreditación, por lo que se puede establecer que su orientación está principalmente en su función de formación de capital humano, investigación, y vinculación con la sociedad, es decir, son universidades orientadas a la función más tradicional, ligada estrechamente al quehacer académico. Sin embargo, uno de estos dos grupos presentaba una rentabilidad sobre el patrimonio que superaba el promedio, esto indica que además de poseer una alta calidad institucional, lograron la generación de excedentes económicos.

Es admisible consignar que en estos dos grupos se encontraron universidades tanto públicas como privadas. Asimismo, se identificaron otros dos grupos de universidades, los que se encuentran bajo el promedio de años de acreditación institucional, situación que muestra que el problema de la calidad de las instituciones de educación superior es una preocupación permanente para las autoridades, más aún en aquellas universidades que como resultado de su quehacer habitual evidencian bajos niveles de calidad y una alta rentabilidad económica. Conllevando esto último a cuestionar la función y el rol que cumplen dichas universidades en la sociedad, aunque claramente se alejan de su función formadora de profesionales de calidad.

En efecto, es posible identificar dos grupos de universidades que poseen una rentabilidad por sobre el promedio, incluso con la presencia de un caso que presenta una rentabilidad del $105 \%$. A este respecto, surge la interrogante en relación con los excedentes generados, situación que lleva a preguntarse dónde estos están siendo reinvertidos, teniendo en cuenta que el lucro está prohibido por ley en Chile, es decir, considerando que estos excedentes no pueden ser retirados por los controladores de dichas instituciones. En este sentido falta avanzar en la regulación y control sobre las empresas vinculadas a las universidades, particularmente respecto de la desviación de excedentes que efectúan las instituciones de educación superior hacia estos.

Las universidades, por tanto, deben velar por la calidad de su quehacer, siendo esta la principal preocupación en torno a la cual orientar su funcionamiento, procesos y resultados. Por ende, su misión y plan estratégico deben estar alineados con el logro y el resguardo de la calidad en la formación de capital humano de excelencia, la investigación y la vinculación con la sociedad, fundamentales en la sociedad del conocimiento, y no hacia la obtención y maximización de excedentes, es decir, a la generación de lucro.

\section{AGRADECIMIENTOS}

Esta investigación cuenta con el patrocinio de los proyectos FONDECYT 1140026 y 1140027 de la Comisión Nacional de Investigación Científica y Tecnológica de Chile.

\section{LIMITACIONES DE LA INVESTIGACIÓN}

Esta investigación contribuye a una primera aproximación en el análisis de la relación entre la rentabilidad y la calidad. Obedece a un contexto histórico determinado, el 2011, donde la discusión referente a la educación superior irrumpió considerablemente en la escena nacional durante 
ese periodo. Ese mismo año, las instituciones de educación superior publicaron información respecto de su patrimonio, esto contribuyó al enriquecimiento del análisis al incorporar el elemento financiero de las instituciones de educación superior. En este sentido se debe clarificar que la información respecto de patrimonio pudo ser obtenida solo para ese año, ya que no se ha vuelto a publicar, lo que limita los resultados de la investigación al no poder desarrollar el análisis de la rentabilidad sobre el patrimonio de las instituciones universitarias para otros años y así profundizar en la validez y confiabilidad de los resultados en este estudio, los que además de ser exploratorios obedecen a un determinado contexto.

De igual modo se señala que la información respecto de la rentabilidad no fue validada por el Ministerio de Educación.

\section{REFERENCIAS}

[1] P. Altbach, L. Reisberg and L. Rumbley. "Trends in global higher education: tracking an academic revolution". Report prepared for the UNESCO World conference on higher education. 2009.

[2] L. Améstica Rivas, H. Gaete Feres y X. Llinas-Audet. "Segmentación y clasificación de las universidades en Chile: desventajas de inicio y efectos de las políticas públicas de financiamiento". Ingeniare. Revista chilena de ingeniería. Vol. $22 \mathrm{~N}^{\circ}$ 3, pp. 384-397. 2014.

[3] E. Anderson and V. Mittal. "Strengthening the satisfaction profit chain". Journal of Service Research. Vol. 3 N$^{\circ}$ 2, pp. 107-120. 2000.

[4] W. Beaver. "Fraud in for profit higher education". Society. Vol. 49 No 3, pp. 274278. 2012.

[5] S. Bjarnason, K. Cheng, J. Fielden, M. Lemaitre, D. Levy and N. Varghese (Eds.) "A new dynamic: Private higher education. Background document prepared for the World Conference on Higher Education". UNESCO. Paris, Francia. 2009.

[6] D. Bok. "Universities in the marketplace: the commercialization of higher education". Princeton University Press. New Jersey, EE.UU. 2003.

[7] J. Brunner y D. Uribe. "Mercados universitarios: el Nuevo escenario de la educación superior". Ediciones Universidad Diego Portales. Santiago, Chile. 2007.

[8] A. Carpenter and C. Bach. "Administrative and academic structures: for-profit and notfor-profit". Kazeroony, H. (Ed.) The Strategic Management of Higher Education, pp. 89108. Business Express Press. Addison, TX. EE.UU. 2011.

[9] Y.C. Cheng. "School effectiveness and improvement in Hong Kong, Taiwan, and mainland China". In B. Creemers and N. Osinga, (Eds), International congress of school effectiveness and school improvement country reports, pp. 11-30. GCO. Netherlands. 1995.

[10] Y.C. Cheng and W.M. Tam. "Multi-models of quality in education". Quality Assurance in Education. Vol. 5 N ${ }^{\circ}$ 1, pp. 22-31. 1997.

[11] J. Daniel, A. Kanwar and S. Uvalić-Trumbić. "Breaking Higher education's Iron Triangle: Access, Cost, and Quality". Change: The Magazine of Higher Learning. Vol. $41 \mathrm{~N}^{\circ} 2$, pp. 30-35. 2009.

[12] N. Fleet. "Movimiento estudiantil y transformaciones sociales en Chile: una perspectiva sociológica". Polis (Santiago). Vol. 10 N $^{\circ}$ 30, pp. 99-116. 2011.

[13] H.A. Giroux. "Democracy's nemesis: The rise of the corporate university". Cultural Studies \$ Critical Methodologies. Vol. 9 No 5, pp. 669-695. 2009.

[14] D. Green (Ed.). "What is quality in higher education?". Taylor \& Francis. Bristol, England. 1994.

[15] A. Gupta. "International Trends and Private Higher Education in India". International Journal of Education Management. Vol. 22 $\mathrm{N}^{\circ}$ 6, pp. 565-594. 2008.

[16] H. Hadi. "Industry partnerships for the sustainability of the higher education institutions: the oum experience". Widyatama International Seminar. 2014.

[17] L. Harvey. "Quality in higher education". Paper at the Swedish Quality Conference. University of Central England in Birmingham. Göteborg, Swedish. 1999.

[18] L. Harvey and D. Green. "Defining quality". Assessment \& evaluation in higher education. Vol. $18 \mathrm{~N}^{\circ}$ 1, pp. 9-34. 1993.

[19] Ø. Helgesen. "Marketing for Higher Education: A Relationship Marketing 
Approach". Journal of Marketing for Higher Education. Vol. $18 \mathrm{~N}^{\circ}$ 1, pp. 50-78. 2008.

[20] O. James. "Predatory Ed: The Conflict Between Public Good and For-Profit Higher Education". Journal of College and University Law. Vol. $38 \mathrm{~N}^{\circ}$ 1, p. 47.2012

[21] L. Jamshidi, H. Arasteh, E. Naveh, H. Zeinabadi and P. Rasmussen. "Developmental patterns of privatization in higher education: a comparative study". Higher Education, pp. 1-15. Springer. Netherlands. 2012.

[22] D. Jarvis. "Regulating higher education: Quality assurance and neo-liberal managerialism in higher education-A critical introduction". Policy and Society. Vol. 33 $\mathrm{N}^{\mathrm{o}}$ 3, pp. 155-166. 2014.

[23] A. Kagumba and G. George. "Quality Assurance Strategy on Organizational Performance: Case of Kenyatta University". European Journal of Business and Management. Vol. 5 No 2, pp. 265-270. 2013.

[24] J. Knight. "Cross-border higher education: issues and implications for quality assurance and accreditation". In Higher Education in the World. UNESCO. Paris, Francia. 2007.

[25] T.L. Keiningham, L. Aksoy and D. Bejou. "How customer lifetime value is changing how business is managed". Journal of Relationship Marketing. Vol. $5 \mathrm{~N}^{\circ} 2 / 3$, pp. 1-6. 2006.

[26] M. Kwiek. "From Growth to Decline? Demand-Absorbing Private Higher Education when Demand is Over". Springer. Dordrecht, Holanda. 2014.

[27] P. Lansing and D. Olsen "The Subsidized Business of Higher Education: An Ethical Analysis of Federal Funding in For-Profit Higher Education". Mustang journal of accounting and finance. Vol. 1, pp. 118-135. 2011.

[28] J. Lavados. "Desafíos pendientes de las políticas públicas en educación superior". Revista calidad en la educación. Vol. 22 $\mathrm{N}^{\circ}$ 1, pp. 23-36. 2005.

[29] D. Levy and W. Zumeta. "Private Higher Education and Public Policy: A Global View". Journal of Comparative Policy Analysis: Research and Practice. Vol. 13 $\mathrm{N}^{\circ}$ 4, pp. 345-349. 2011.

[30] D. Levy. "Public Policy for Private Higher Education: A Global Analysis". Journal of
Comparative Policy Analysis: Research and Practice. Vol. 13 No 4, pp. 383-396. 2011.

[31] D. Levy. "For-profit vis-à-vis nonprofit private higher education". International Higher education. $\mathrm{N}^{\circ}$ 54, pp. 12-13. 2009.

[32] C. Lim. "Australian transnational higher education. Quality assurance in Singapore and Malaysia". Thesis for the degree of Doctor of philosophy. Deakin University. Australia. 2011.

[33] B.J. Mandernach, H. Radda, S. Greenberger and K. Forrest. "Challenging the Status Quo: The Influence of Proprietary Learning Institutions on the Shifting Landscape of Higher Education". In Transformative Perspectives and Processes in Higher Education, pp. 31-48. Springer International Publishing. 2015.

[34] S. Marginson. "The public/private divide in higher education: A global revision". Higher Education. Vol. $53 \mathrm{~N}^{\circ}$ 3, pp. 307-333. 2007.

[35] K. Mok. "The growing importance of the privateness in education: challenges for higher education governance in China". Compare: A Journal of Comparative and International Education. Vol. $39 \mathrm{~N}^{\circ} 1$, pp. 35-49. 2009.

[36] D. Mungu. "Is market orientation a relevant strategy for higher education institutions?". International Journal of Quality and Service Sciences. Vol. $1 \mathrm{~N}^{\mathrm{o}}$ 3, pp. 311-333. 2009.

[37] M. Muñoz y C. Blanco. "Una taxonomía de las universidades chilenas". Calidad en la educación. Vol. 38, pp. 181-213. 2013.

[38] H. Nadiri, J. Kandampully and K. Hussain. "Students' perceptions of service quality in higher education". Total Quality Management \& Business Excellence. Vol. $20 \mathrm{~N}^{\circ}$ 5, pp. 523535. 2009.

[39] OCDE. "Revisión de políticas nacionales de educación. La educación superior en Chile". OCDE y BIRD/Banco Mundial. Santiago, Chile. 2009.

[40] G. Odhiambo. "Quality assurance for public higher education: context, strategies and challenges in Kenya". Higher Education Research \& Development, (ahead-of-print), pp. 1-14. 2014.

[41] G. Odhiambo. "Higher education quality in Kenya: a critical reflection of key challenges". Quality in Higher Education. Vol. $17 \mathrm{~N}^{\mathrm{o}} 3$, pp. 299-315. 2011. 
[42] M. Oketch. "Public-private mix in the provision of higher education in East Africa: Stakeholders' perceptions". Compare: A Journal of Comparative and International Education. Vol. $39 \mathrm{~N}^{\circ}$ 1, pp. 21-33. 2009.

[43] L.O. Oyarzún. "Desarrollo es acceso a la educación: las movilizaciones estudiantiles en Chile". Cambio de ciclo: crisis, resistencias y respuestas globales, pp. 221-234. 2012.

[44] M. Pachuashvili. "Governmental Policies and their Impact on Private Higher Education Development in Post-Communist Countries: Hungary, Latvia, Lithuania and Georgia, 1990-2005". Journal of Comparative Policy Analysis: Research and Practice. Vol. 13 $\mathrm{N}^{\mathrm{o}}$ 4, pp. 397-410. 2011.

[45] L. Pedraja Rejas y E. Rodríguez Ponce. "Dirección estratégica y gestión en instituciones de Educación Superior". En A. Bernasconi (Ed.). "La educación superior en Chile. Transformación, desarrollo y crisis". Colección de Estudios en Educación superior. CEPPE. Ediciones UC. 475-516. 2014

[46] L. Pedraja Rejas. "Proyecto Estratégico Institucional y la Calidad de las Universidades: Un Estudio desde Chile". Tesis Doctoral en Ciencias de la Educación de la Pontificia Universidad Católica de Chile. Facultad de Educación. Pontificia Universidad Católica de Chile, pp. 269. Santiago, Chile. 2014.

[47] E. Rodríguez-Ponce. "El rol de la universidades en la sociedad del conocimiento y en la era de la globalización: Evidencia desde Chile". Interciencia. Vol. $34 \mathrm{~N}^{\circ}$ 11, pp. 822-829. 2009.

[48] E. Rodríguez-Ponce, N. Fleet y M. Delgado. "La acreditación en la generación de información sobre la calidad de la educación superior". Calidad en la Educación. Vol. 31, pp. 212-230. 2009.

[49] B.C. Sanyal and D.B. Johnstone. "International trends in the public and private financing of higher education". Prospects. Vol. $41 \mathrm{~N}^{\mathrm{o}} 1$, pp. 157-175. 2011.

[50] M. Shah and C. Nair. "Engaging with Quality: Quality Assurance and Capacity Building in Private Higher Education". Proceedings of the Australian Quality Forum. 2011.

[51] M. Shah and G. Brown. "The Rise of Private Higher Education in Australia: Maintaining Quality Outcomes and Future Challenges". In Proceedings of the Australian Universities Quality Forum (AUQF), pp. 143-150. Australian Universities Quality Agency. Melbourne, Australia. 2009.

[52] S. Schwarz and D. Westerheijden D. (Eds) "Accreditation and Evaluation in the European Higher Education Area". Springer. Dordrecht, Holanda. 2005.

[53] Secretaría General Consejo de Rectores Universidades Chilenas. "Nueva legislación universitaria chilena”. Secretaría General del Consejo de Rectores. Santiago, Chile. 1981.

[54] W. Tierney and V. Lechuga. "Differences in academic work at traditional and forprofit postsecondary institutions. Policy implications for academic freedom". In G. Hentschke, V. Lechuga and W. Tierney (Eds.). "For -profit colleges and universities. Their markets, regulation, performance and place in higher education", pp. 71-90. Stylus Publishing. Virginia, EE.UU. 2010.

[55] J.Tilak. "Private higher education: philanthropy to profits. A: Higher education in the world 2006: the financing universities", pp. 113-121. GUNI. Barcelona, España. 2006. 